\title{
CDISC SDTM Submission Domain Abbreviation Terminology
}

National Cancer Institute

\section{Source}

National Cancer Institute. CDISC SDTM Submission Domain Abbreviation Terminology. NCI Thesaurus. Code C66734.

Terminology codelist used with Submission Domain Abbreviation within the Clinical Data Interchange Standards Consortium Study Data Tabulation Model. 